\title{
CHANDRA OBSERVATIONS OF CYGNUS A: MAGNETIC FIELD STRENGTHS IN THE HOT SPOTS OF A RADIO GALAXY
}

\author{
A. S. Wilson ${ }^{1}$ AND A. J. Young \\ Astronomy Department, University of Maryland, College Park, MD 20742; wilson@astro.umd.edu, ayoung@astro.umd.edu \\ AND \\ P. L. SHOPBELL \\ Department of Astronomy, California Institute of Technology, MS 105-24, Pasadena, CA 91125; pls@astro.caltech.edu \\ Received 2000 July 28; accepted 2000 September 18; published 2000 November 8
}

\begin{abstract}
We report X-ray observations of the powerful radio galaxy Cygnus A with the Chandra X-Ray Observatory. This Letter focuses on the radio hot spots, all four of which are detected in X-rays with a very similar morphology to their radio structure. X-ray spectra have been obtained for the two brighter hot spots (A and D). Both are well described by a power law with photon index $\Gamma=1.8 \pm 0.2$ absorbed by the Galactic column in the direction of Cygnus A. Thermal X-ray models require too high gas densities and may be ruled out. The images and spectra strongly support synchrotron self-Compton models of the X-ray emission, as proposed by Harris, Carilli, \& Perley on the basis of ROSAT imaging observations. Such models indicate that the magnetic field in each of the brighter hot spots is $1.5 \times 10^{-4} \mathrm{G}$, with an uncertainty of a few tens of percent. This value is close to the equipartition field strengths assuming no protons are present. The possibility that the X-rays are synchrotron radiation is briefly discussed but not favored. We speculate that production of the $\gamma \sim 10^{7}$ electrons necessary for X-ray synchrotron radiation from hot spots is inhibited when the external gas density is high, as is the case when the radio galaxy is within a cooling flow.
\end{abstract}

Subject headings: galaxies: active — galaxies: individual (Cygnus A) — galaxies: jets — galaxies: nuclei — magnetic fields - X-rays: galaxies

\section{INTRODUCTION}

The spectral emissivity of optically thin synchrotron radiation is proportional to $n_{e 0} B^{(p+1) / 2}$, where $n_{e 0}$ is the constant in the relativistic electron number density spectrum $\left[n_{e}(\gamma) d \gamma=\right.$ $\left.n_{e 0} \gamma^{-p} d \gamma, \gamma=E / m_{e} c^{2}\right]$ and $B$ is the magnetic field strength. Observations of synchrotron sources allow this product to be determined, but not $n_{e 0}$ and $B$ separately. It is common practice to quote the equipartition magnetic field $B_{\text {eq }}$, the field for which the energies in relativistic particles and magnetic field are equal; this field is close to that at which the total energy in relativistic particles plus magnetic field is a minimum. This inability to obtain a direct measurement of the magnetic field strength has seriously hampered progress in understanding cosmic synchrotron sources.

The relativistic electrons will inevitably scatter any photons in the source through the inverse Compton process. The emissivity of inverse Compton radiation depends on the electron energy spectrum and the radiation spectrum, the latter being an observable quantity for an isotropic source. Detection of inverse Compton radiation thus permits $n_{e 0}$ to be measured and hence $B$ from the synchrotron spectrum. If the photons being scattered are the synchrotron radiation itself, the resulting emission is called synchrotron self-Compton (SSC) radiation.

For a typical extended nonthermal radio source, much of the inverse Compton emission is expected to be radiated at X-ray energies. Unfortunately, the existence of other mechanisms that generate X-rays - thermal and synchrotron emissions - and the poor spatial and spectral resolutions of previous X-ray observatories has precluded the use of this method to measure magnetic field strengths in either supernova remnants or extragalactic radio sources.

\footnotetext{
${ }^{1}$ Adjunct Astronomer, Space Telescope Science Institute, 3700 San Martin Drive, Baltimore, MD 21218; awilson@ stsci.edu.
}

In this Letter, we present the first results of a study of the nearby $(z=0.0562)$, powerful radio galaxy Cygnus A with the Chandra X-Ray Observatory. We focus on the X-ray emission of the radio hot spots. Previously, Harris, Carilli, \& Perley (1994, hereafter HCP) detected soft X-ray emission from the dominant western and eastern hot spots (termed A and D by Hargrave \& Ryle 1974) with the High Resolution Imager of the ROSAT observatory. They compared the measured X-ray fluxes with predictions of SSC models and argued that the magnetic fields in the hot spots are quite close to the equipartition values, calculated assuming no contribution from relativistic protons. The angular resolution of the ROSAT HRI was $\simeq 5^{\prime \prime}$, so the point-spread function included both weaker radio structures (such as the fainter hot spots B and E; see Perley, Dreher, \& Cowan 1984) and thermal emission from the cluster gas. More significantly, the detector had almost no spectral resolution, so HCP were unable to measure the X-ray spectra of the hot spots. The Chandra observations overcome these limitations. The subarcsecond angular resolution and high sensitivity of Chandra have allowed detection and spatial resolution of X-ray emission from all four hot spots. We also report the X-ray spectra of the two brightest hot spots (A and D), finding excellent agreement with the predictions of the SSC model. This agreement strongly favors the SSC model, but does not rule out a contribution from X-ray synchrotron emission, as we briefly discuss. We use $H_{0}=50 \mathrm{~km} \mathrm{~s}^{-1} \mathrm{Mpc}^{-1}$ and $q_{0}=0$ throughout. Other aspects of the Chandra results on Cygnus A will be discussed elsewhere.

\section{OBSERVATIONS AND REDUCTION}

Cygnus A was observed by the Chandra X-Ray Observatory on 2000 May 21 (sequence number 700032, obsid 360) using the Advanced CCD Imaging Spectrometer spectroscopic array. The nucleus was centered $20^{\prime \prime}$ in the $-Y$-direction from the 


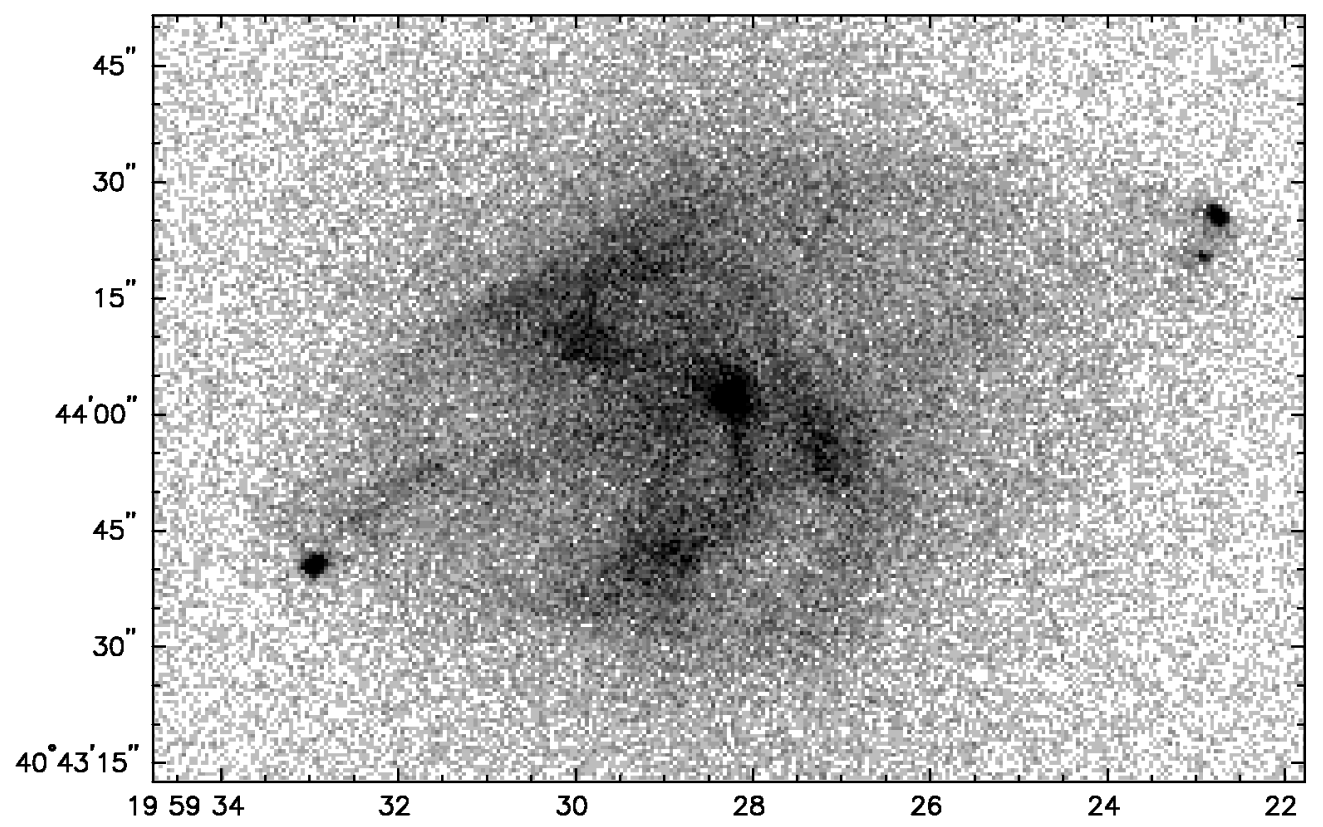

FIG. 1.-Gray-scale representation of the Chandra X-ray image of Cygnus A. The shading is proportional to the square root of the intensity. Coordinates are for epoch J2000.0 both here and in Fig. 2.

location of best focus on chip S3. All of the regions of radio emission from Cyg A were imaged on S3. The total good time interval was $34.7 \mathrm{ks}$ taken with the default frame time of $3.2 \mathrm{~s}$. The data extraction and analysis have been performed using version 1.1.3 of the CIAO software and version 11.0 of XSPEC. A new level 2 events file, with the correct gain map (acisD2000-01-29gainN0001.fits), was made from the events file supplied by the Chandra Science Center. The data were inspected for bad aspect and high background times, but none were found. The response matrix file and ancillary response file were taken from calibration data obtained with the chip at $-120^{\circ} \mathrm{C}$ (the temperature during the observations). The counts used to obtain the spectra of hot spots A and D were taken from circular regions of radii 2".3 and 2".2, respectively, with background taken from annular regions of width $\simeq 2$ " concentric with the source region.

\section{RESULTS}

\subsection{Morphology}

Figure 1 is a gray-scale plot of the $\mathrm{X}$-ray emission from the region of the radio source. The Chandra astrometry shows that the nuclear X-ray source agrees with the nuclear radio source to within 1"4. When the X-ray and radio nuclei are aligned, the compact X-ray sources at the southeast and northwest edges of Figure 1 coincide with the corresponding radio hot spots to within 0 ".5. Figure 2 shows X-ray contours on a gray scale of a $6 \mathrm{~cm}$ image with resolution 0".35 (Perley et al. 1984; Carilli
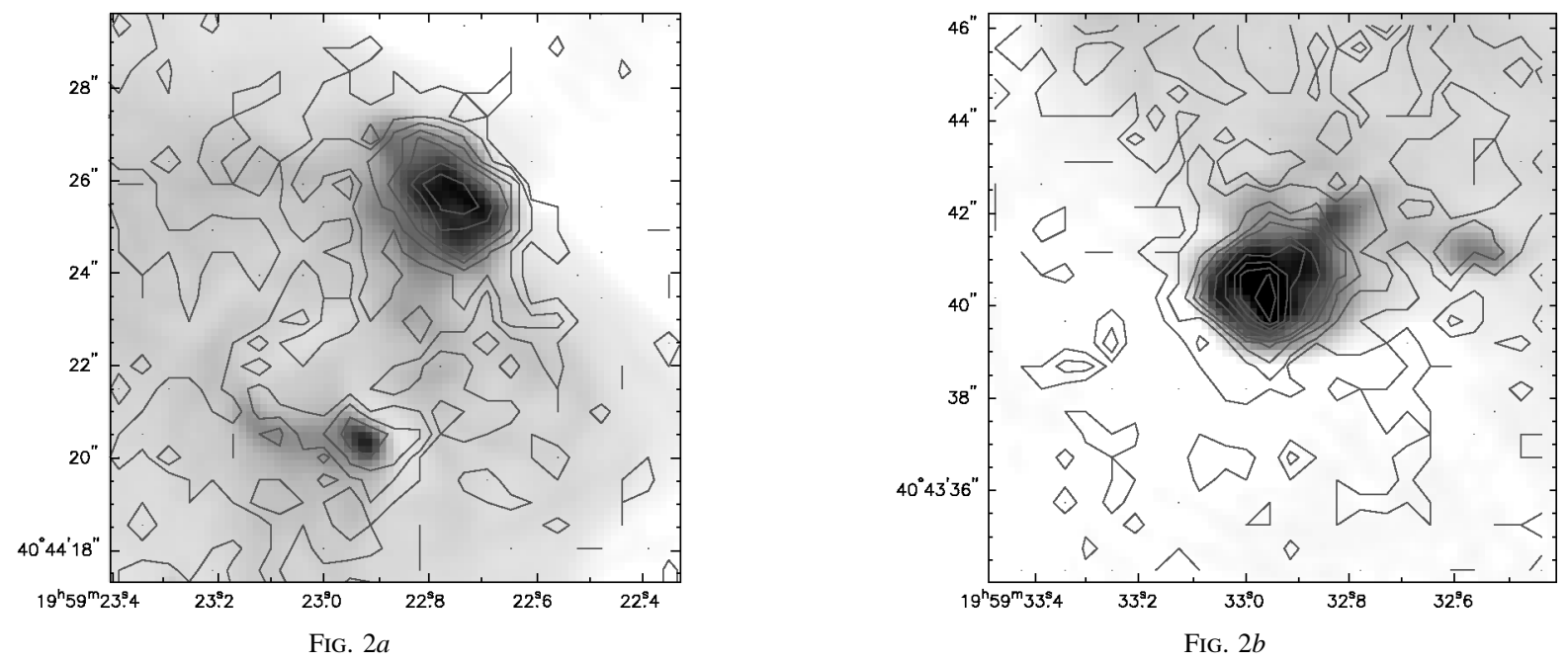

FIG. 2.-(a) X-ray emission (contours) superposed on a $6 \mathrm{~cm}$ Very Large Array radio map (gray scale) of the region of the western hot spots (A, the brighter, and $\mathrm{B}, \simeq 6^{\prime \prime}$ southeast of $\left.\mathrm{A}\right)$. Contours are plotted at $2,4,8,12,16,24$, and 32 counts pixel ${ }^{-1}\left(00^{\prime \prime} .5 \times 0.5\right)$. The gray scale is proportional to the square root of the radio brightness. (b) Same as Fig. $2 a$, but for the eastern hot spots (D, the brighter, and E, $\simeq 4$ " west of D). Contours are plotted at 2, 4, 8, 12, 16, 24, 32, 40,48 , and 56 counts pixel ${ }^{-1}$. 
TABLE 1

Spectral Fits to the X-Ray Emission of the Hot Spots ${ }^{\mathrm{a}}$

\begin{tabular}{lcccc}
\hline \hline Hot Spot & $N_{\mathrm{H}}{ }^{\mathrm{b}}$ & $\Gamma$ & Normalization $^{\mathrm{c}}$ & $\begin{array}{c}\chi^{2} \\
\text { (degrees of freedom) }\end{array}$ \\
\hline A $\ldots \ldots .$. & $3.26_{-0.61}^{+0.74}$ & $1.84_{-0.19}^{+0.22}$ & $2.93_{-0.63}^{+0.81}$ & $27(27)$ \\
D $\ldots \ldots .$. & $3.30_{-0.26}^{+0.63}$ & $1.75_{-0.17}^{+0.19}$ & $4.40_{-0.77}^{+0.97}$ & $31(39)$ \\
\hline
\end{tabular}

${ }^{a}$ Errors are $90 \%$ confidence for single parameter of interest.

${ }^{\mathrm{b}}$ In units of $10^{21}$ atoms $\mathrm{cm}^{-2}$.

${ }^{\mathrm{c}}$ In units of $10^{-5}$ photons $\mathrm{keV}^{-1} \mathrm{~cm}^{-2} \mathrm{~s}^{-1}$ at $1 \mathrm{keV}$.

\& Barthel 1996) in the vicinities of the western (A and B) and eastern (D and E) hot spots.

As may be seen in Figure 2, the extents and morphologies of the X-ray and radio hot spots are very similar, with the directions of elongation agreeing to within a few degrees. Despite the somewhat lower resolution of the X-ray image, it is clear that the X-ray emission comes from essentially the same region as the radio emission in each hot spot.

\subsection{Spectra}

Hot spots $\mathrm{B}$ and $\mathrm{E}$ are too weak to obtain reliable X-ray spectra, so we focus on A and D. The results of modeling each spectrum with an absorbed power law are shown in Table 1. In both cases, the absorbing column is $N_{\mathrm{H}}=3.3 \times 10^{21} \mathrm{~cm}^{-2}$, in excellent agreement with the Galactic column $\left(3.3 \times 10^{21} \mathrm{~cm}^{-2}\right.$; $\mathrm{HCP})$ in the direction of Cygnus A. The photon indices are similar at $\Gamma=1.8 \pm 0.2$. Alternatively, the spectra may be well described by a Raymond-Smith thermal plasma model with temperatures $4.9 \mathrm{keV}$ (A) and $6.0 \mathrm{keV}$ (D).

\subsection{X-Ray Emission Mechanism}

A thermal model requires a density of $0.5 \mathrm{~cm}^{-3}$ in each hot spot. This value is $10^{3}$ times larger than the upper limit to the internal density in the hot spots from the absence of Faraday depolarization (Dreher, Carilli, \& Perley 1987). It is also hard to understand how such a high density could be produced in the hot spots given that the density of the intracluster medium near them is only $\simeq 0.01 \mathrm{~cm}^{-3}$ (HCP; Reynolds \& Fabian 1996).
TABLE 2

Parameters of Synchrotron Self-Compton Models

\begin{tabular}{lcccccc}
\hline \hline Hot Spot & $\begin{array}{c}B \\
\left(\times 10^{-4} \mathrm{G}\right)\end{array}$ & $\begin{array}{c}n_{e 0} \\
\left(\mathrm{~cm}^{-3}\right)\end{array}$ & $\alpha$ & $\begin{array}{c}\gamma_{\text {break }} \\
\left(\times 10^{3}\right)\end{array}$ & $\begin{array}{c}\gamma_{\max } \\
\left(\times 10^{4}\right)\end{array}$ & $\begin{array}{c}\text { Radius } \\
(\mathrm{kpc})\end{array}$ \\
\hline A $\ldots \ldots$. & 1.5 & $1.9 \times 10^{-3}$ & 0.55 & 3.5 & 5.0 & 2.0 \\
$\mathrm{D} \ldots \ldots$. & 1.5 & $5.5 \times 10^{-4}$ & 0.50 & 5.5 & 5.0 & 2.2 \\
\hline
\end{tabular}

We conclude that a thermal model for the X-ray emission of the hot spots is untenable.

In view of the success of an SSC model in reproducing the intensity of the soft X-ray emission (HCP), it is natural to check whether the model can also reproduce the X-ray spectra. From the radio spectra of the hot spots (Carilli et al. 1991), we first calculated the internal radiant energy density in the hot spots, modeled as uniformly emitting spheres, from $\epsilon_{R}=3 L_{R} R / 4 \mathrm{cV}$, where $L_{R}$ is the total radio luminosity, $R$ and $V$ are the radius and volume of the hot spot, and $c$ is the speed of light. The results are $\epsilon_{R} \simeq 3 \times 10^{-11} \mathrm{ergs} \mathrm{cm}^{-3}$ for each hot spot. These values are $\simeq 100$ times larger than the energy density of the microwave background $\left(\epsilon_{M} \simeq 4 \times 10^{-13} \mathrm{ergs} \mathrm{cm}^{-3}\right)$, showing that an SSC model is indeed appropriate. The radiant energy densities in the hot spots are, however, $\simeq 100$ times smaller than that in the magnetic field $\left(\epsilon_{B} \simeq 3 \times 10^{-9} \mathrm{ergs} \mathrm{cm}^{-3}\right)$ assuming equipartition and no relativistic protons. Thus, the rate of energy loss by the electrons to synchrotron radiation will be $\simeq 100$ times larger than to SSC radiation.

To calculate the SSC spectra, we have used the code of Band \& Grindlay (1985, 1986), which assumes spherical geometry. Following Carilli et al. (1991), the radio spectrum of each hot spot was modeled as a broken power law with spectral index $\alpha=0.55$ (hot spot $\mathrm{A} ; S \propto \nu^{-\alpha}$ ) and 0.50 (hot spot D) below the break frequency and 1.05 (A) and 1.0 (D) above it. Such a change in slope of 0.5 is expected in models of continuous injection of electrons accompanied by synchrotron losses (Kardashev 1962). The emitting region was taken to be a uniform sphere in each case.

The results of the modeling are given in Table 2 and compared with the Chandra spectra in Figures $3 a$ and $3 b$. As may be seen, the predicted SSC radiation is in excellent agreement

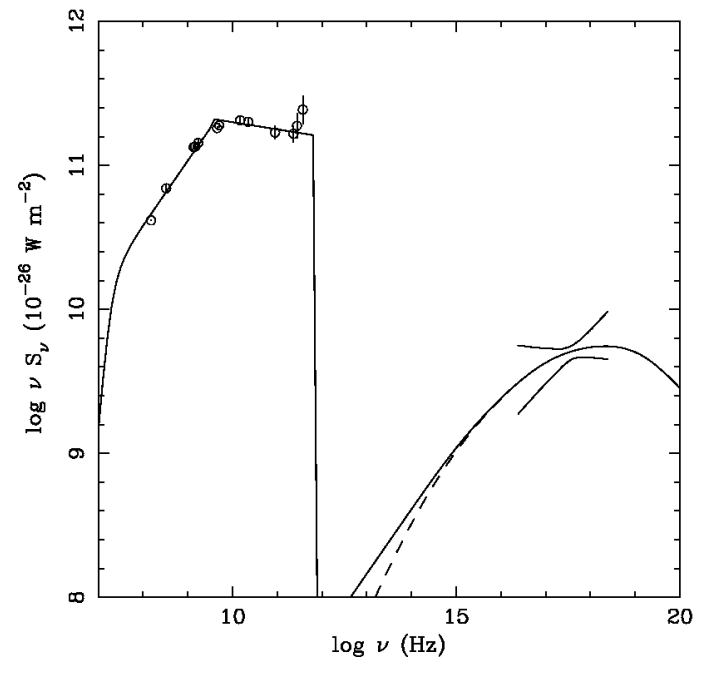

FIG. $3 a$

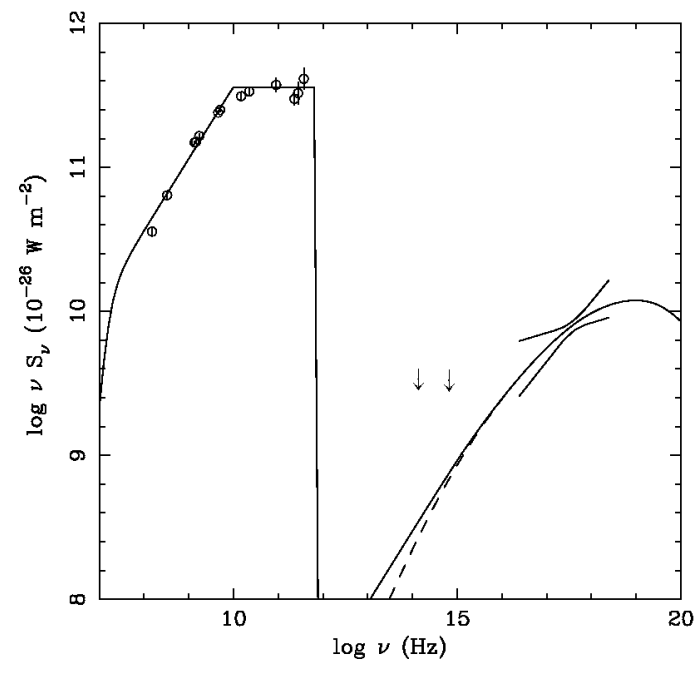

FIG. $3 b$

FIG. 3.-(a) Spectrum of hot spot A. The points show the radio fluxes and the line through them the model of the synchrotron radiation. The "bow tie" is the Chandra-measured boundary of the X-ray spectrum (these error lines are $90 \%$ confidence after freezing $N_{\mathrm{H}}$ at its best-fit value, which coincides with the Galactic column). The solid line is the predicted SSC spectrum for $\gamma_{\min }=1$, and the dashed line is for $\gamma_{\min }=100$. (b) Same as Fig. $3 a$, but for hot spot D. The nearinfrared and optical upper limits are from Meisenheimer, Yates, \& Röser (1997), including their allowance for obscuration. 
with the Chandra-observed spectrum for a magnetic field of $1.5 \times 10^{-4} \mathrm{G}$ in each hot spot, in good agreement with the results of HCP based on the X-ray intensity. This value may be compared with the equipartition values of $2.8 \times 10^{-4} \mathrm{G}$ (hot spot A) and $2.5 \times 10^{-4} \mathrm{G}$ (hot spot $\mathrm{D}$ ), calculated assuming no relativistic protons, the broken power-law spectra of Carilli et al. (1991), low-frequency cutoffs at $10 \mathrm{MHz}$, and highfrequency cutoffs at $400 \mathrm{GHz}$. The calculated field is insensitive to the precise cutoff frequencies. The SSC model predicts spectral steepening toward higher energies within the Chandra band. We have searched for this effect, finding a hint of a larger value of $\Gamma$ in the $2.5-6 \mathrm{keV}$ band than in the $0.7-2.5 \mathrm{keV}$ band for hot spot D. However, this difference in photon indices is not significant.

Uncertainty in the magnetic field obtained with the SSC model results from a number of factors, including (1) the idealization of the hot spots as uniform spheres of known radii, (2) the errors in the Chandra-measured spectra, and (3) modeling the radio spectrum of each hot spot as a broken power law, with sharp changes in the slopes of both the electron energy and the synchrotron radiation spectra at the break energy and frequency. Comparison of the formulae for synchrotron and inverse Compton radiation indicates that changing the volume by a factor of 2 changes $B$ by $\sim 30 \%$. The normalization of the Chandra spectra are uncertain by $\simeq 17 \%-28 \%$ (Table 1). An error of $25 \%$ in SSC flux changes $B$ by $\simeq 15 \%$. Lastly, the idealized treatment of the electron energy and synchrotron spectra around the break is estimated to contribute $\leqslant 10 \%$ uncertainty. The precise locations and shapes of the high-energy cutoffs have little effect on the SSC spectra in the Chandra band. We conclude that the error in the magnetic fields derived from the SSC model amounts to a few tens of percent.

\section{CONCLUDING REMARKS}

The X-ray spectra of the two brightest radio hot spots (A and D) of Cygnus A are in excellent agreement with an SSC model in which the magnetic field is $1.5 \times 10^{-4} \mathrm{G}$. This value is close to the equipartition fields of $2.8 \times 10^{-4}$ and $2.5 \times$ $10^{-4} \mathrm{G}$ for hot spots $\mathrm{A}$ and $\mathrm{D}$, respectively, calculated assuming no relativistic protons are present (i.e., the ratio of the energy density in relativistic protons to that in electrons, $K=0$ ). The most straightforward interpretation is that the relativistic gas is an electron-positron plasma and is close to equipartition with the magnetic field. If, on the other hand, there is significant energy in relativistic protons (e.g., $K \sim 100$, a value appropriate to the relativistic protons and electrons observed at the top of the Earth's atmosphere) and the X-rays are still SSC radiation, the energy density in relativistic protons must exceed that in the magnetic field. It is notable that the magnetic field cannot be less than $1.5 \times 10^{-4} \mathrm{G}$ since the SSC radiation would then exceed the observed $\mathrm{X}$-radiation.

The alternative is that $B>1.5 \times 10^{-4} \mathrm{G}$, in which case the predicted SSC emission would be too weak to account for the observed X-ray emission. The X-rays would then have to be synchrotron radiation, as we have recently suggested for the jet and hot spot of Pictor A (Wilson, Young, \& Shopbell 2001). Synchrotron X-ray emitting electrons in magnetic fields of hundreds of microgauss strength have synchrotron half-lives of order years. They must thus be continuously accelerated within the hot spots. Here, again, there are two options-direct electron acceleration to $\gamma \simeq 10^{7}-10^{8}$ or a "proton-induced cascade." In the latter process (e.g., Mannheim, Krülls, \& Biermann 1991; Biermann 1996), ultrahigh energy protons $\left(\gamma_{p} \sim\right.$ $10^{11}$ ), perhaps shock-accelerated, interact with photons to initiate a cascade, which eventually yields relativistic electronpositron pairs. The peak of the radiation from this process is at $\sim \mathrm{MeV}$ energies, and the spectral index predicted in the Chan$d r a$ band is $\alpha \simeq 0.7$ (Mannheim et al. 1991), in agreement with the spectral index observed. Observations at higher energies than the Chandra band, where the proton-induced cascade predicts a harder spectrum than the SSC model, are needed to distinguish the two processes.

$\mathrm{X}$-ray emission has now been detected from a number of hot spots in radio galaxies. For three galaxies-3C 123 (Hardcastle, Birkinshaw, \& Worrall 2000), 3C 295 (Harris et al. 2000), and Cygnus A (this Letter) - the X-ray emission is well explained by SSC emission from the radio synchrotron-emitting electrons with a magnetic field close to equipartition for $K=0$. In three other galaxies-Pictor A (Wilson et al. 2001), 3C 120 (Harris et al. 1999), and 3C 390.3 (Harris, Leighly, \& Leahy 1998) - the $\mathrm{X}$-ray emission is orders of magnitude too strong to be SSC emission with an equipartition magnetic field. Furthermore, the $\mathrm{X}$-ray spectrum of Pictor A predicted by the simplest SSC model disagrees with that observed. In these cases, the X-rays may be synchrotron radiation from $\gamma \sim 10^{7}$ electrons. It is notable that 3C 295 and Cygnus A are in clusters with prominent cooling flows, while 3C 123 is in a cluster with strong, extended X-ray emission and thus may be within a cooling flow. In contrast, Pictor A, 3C 120, and 3C 390.3 are not in cooling flows. It is tempting to speculate that the presence of high-density surrounding gas may inhibit production of X-ray synchrotron-emitting electrons in hot spots. Several processes may be relevant to this effect, e.g., hot spots propagating into the dense gas of a cooling flow should have lower outward velocities, and there may be more entrainment of thermal gas into the jet and hot spot. Such effects may reduce the efficiency with which $\gamma \sim 10^{7}$ electrons are accelerated. Future Chandra X-ray observations of additional radio galaxies should shed light on these issues.

We are grateful to Rick Perley for providing the VLA $6 \mathrm{~cm}$ map of Cygnus A in numerical form. We also wish to thank the staff of the Chandra Science Center, especially Dan Harris and Shanil Virani, for their help. This research was supported by NASA grant NAG-81027 and by the Graduate School of the University of Maryland.

\section{REFERENCES}

Band, D. L., \& Grindlay, J. E. 1985, ApJ, 298, 128 1986, ApJ, 308, 576

Biermann, P. L. 1996, in Cygnus A-Study of a Radio Galaxy, ed. C. L. Carilli \& D. E. Harris (Cambridge: Cambridge Univ. Press), 139

Carilli, C. L., \& Barthel, P. D. 1996, A\&A Rev., 7, 1

Carilli, C. L., Perley, R. A., Dreher, J. W., \& Leahy, J. P. 1991, ApJ, 383, 554

Dreher, J. W., Carilli, C. L., \& Perley, R. A. 1987, ApJ, 316, 611

Hardcastle, M. J., Birkinshaw, M., \& Worrall, D. M. 2000, MNRAS, submitted Hargrave, P. J., \& Ryle, M. 1974, MNRAS, 166, 305

Harris, D. E., Carilli, C. L., \& Perley, R. A. 1994, Nature, 367, 713 (HCP)
Harris, D. E., Hjorth, J., Sadun, A. C., Silverman, J. D., \& Vestergaard, M. 1999, ApJ, 518, 213

Harris, D. E., Leighly, K. M., \& Leahy, J. P. 1998, ApJ, 499, L149

Harris, D. E., et al. 2000, ApJ, 530, L81

Kardashev, N. S. 1962, Soviet Astron.-AJ, 6, 317

Mannheim, K., Krülls, W. M., \& Biermann, P. L. 1991, A\&A, 251, 723

Meisenheimer, K., Yates, M. G., \& Röser, H.-J. 1997, A\&A, 325, 57

Perley, R. A., Dreher, J. W., \& Cowan, J. J. 1984, ApJ, 285, L35

Reynolds, C. S., \& Fabian, A. C. 1996, MNRAS, 278, 479

Wilson, A. S., Young, A. J., \& Shopbell, P. L. 2001, ApJ, in press 